\title{
Lymph Node Ratio is an Important Prognostic Factor in Curatively Resected Gallbladder Carcinoma, Especially in Node-positive Patients: An Experience from Endemic Region in a Developing Country
}

\author{
Shah Naveed ${ }^{1}$, Hasina Qari ${ }^{2}$, Cao M Thau $^{3}$, Pipit Burasakarn ${ }^{4}$, Abdul W Mir $^{5}$, Brij Bhushan Panday ${ }^{6}$
}

\section{Abstract}

Background: Metastasis to lymph nodes is a bad prognostic factor in patients with gallbladder carcinoma who undergo radical cholecystectomy. During the past decade, studies have brought focus on lymph node ratio (LNR) as an additional valuable prognostic factor in these cases.

Our research studied the factors that predicted the recurrence of disease and survival of patients with gallbladder carcinoma who were treated with surgical resection, concentrating especially on the lymph nodal status as a prognostic factor and LNR in node-positive T1-T3 cases.

Methods: In our hospital, Mahavir Cancer Institute and Research Centre, we reviewed retrospective data, from 2009 to 2014 , of 60 patients who had undergone radical cholecystectomy for gallbladder carcinoma. We staged the patients as per the AJCC eight edition. Predictive factors that affect disease-free survival (DFS), like age, gender, postoperative complications, lymphovascular invasion (LVI) and perineural invasion (PVI), lymph node dissection, differentiation, T stage, N stage, number of lymph nodes involved, and LNR, were examined statistically.

Results: Lymph nodal involvement was found to be a principal predictive factor in cases in whom radical cholecystectomy was done. The number of lymph nodes dissected determined the prognosis in NO cases. LNR was a strong prognostic factor for DFS in cases of curatively resected gallbladder cancer.

Conclusion: LNR is a strong predictive factor in radically resected gallbladder carcinoma cases.

Keywords: Gallbladder carcinoma, Lymph nodal ratio, Lymph nodes.

Euroasian Journal of Hepato-Gastroenterology (2021): 10.5005/jp-journals-10018-1336
\end{abstract}

\section{INTRODUCTION}

Gallbladder carcinoma is a very aggressive malignancy with a dismal prognosis and about 5 -year survival. ${ }^{1}$ The only chance for long-term survival is radical surgery mainly if patients are operated on in the early stage. In patients with resected gallbladder cancer, the presence of metastases in lymph nodes is an essential prognostic factor. ${ }^{2,3}$ Those cases who have metastases in lymph nodes have poor survival than those patients who do not have any nodal metastases. ${ }^{4}$ Radical lymph node dissection in gallbladder carcinoma has shown improved survival, and even some patients had long survival as reported in the literature with some cases of improved survival with nodal disease. ${ }^{5,6}$ Thus, nodal dissection has a crucial part in the treatment of gallbladder cancer.

An insufficient number of lymph nodes dissected decreases survival and also falsely under stage of many gastrointestinal and biliary cancers. ${ }^{7}$ As is evident from the literature on gastrointestinal cancers, the total number of lymph nodes dissected had a bearing on survival as there is decreased survival if the aggregate of lymph nodes dissected is less. ${ }^{8}$ In various gastrointestinal malignancies, the LNR between involved and total number of nodes dissected has been demonstrated as an important prognostic factor. ${ }^{9,10}$

In their paper, Choi et al. described that LNR is a significant independent prognostic factor in gallbladder carcinoma patients who underwent radical cholecystectomy. ${ }^{11}$ It is still not apparent if the LNR can replace lymph node count in the AJCC staging system. The new edition of the AJCC staging uses a different indicator of
${ }^{1}$ Department of Surgical Oncology, Upper GI and HPB Oncosurgery, SKIMS, Srinagar, Jammu and Kashmir, India

${ }^{2}$ Department of Health and Family Welfare, Jammu and Kashmir, India ${ }^{3}$ HPB Division, Institute of Gastroenterology, Tokyo Womens Medical University Hospital, Tokyo, Japan

${ }^{4}$ Department of Surgery, Phramongkutklao Hospital, Bangkok, Thailand

${ }^{5}$ Department of Surgical Oncology, SKIMS, Srinagar, Jammu and Kashmir, India

${ }^{6}$ Department of Surgical Oncology, Mahavir Cancer Sansthan, Patna, Bihar, India

Address reprint requests to: Shah Naveed, Department of Surgical Oncology, Upper GI and HPB Oncosurgery, SKIMS, Srinagar, Jammu and Kashmir, India, email: kingshahnaveed@yahoo.co.in

How to cite this article: Naveed S, Qari H, Thau CM, et al. Lymph Node Ratio is an Important Prognostic Factor in Curatively Resected Gallbladder Carcinoma, Especially in Node-positive Patients: An Experience from Endemic Region in a Developing Country. Euroasian J Hepato-Gastroenterol 2021;11(1):1-5.

Source of support: Nil

Conflict of interest: None

the $\mathrm{N}$ stage formed on the number of nodes involved in place of the location of involved nodes in the seventh edition. ${ }^{12}$

In view of the data reported above, our aim was to point out the factors that would predict cancer recurrence and also predict

(c) The Author(s). 2021 Open Access This article is distributed under the terms of the Creative Commons Attribution 4.0 International License (https://creativecommons. org/licenses/by-nc/4.0/), which permits unrestricted use, distribution, and non-commercial reproduction in any medium, provided you give appropriate credit to the original author(s) and the source, provide a link to the Creative Commons license, and indicate if changes were made. The Creative Commons Public Domain Dedication waiver (http://creativecommons.org/publicdomain/zero/1.0/) applies to the data made available in this article, unless otherwise stated. 
survival of gallbladder carcinoma patients at our institute who had undergone radical cholecystectomy, concentrating mainly on the prognostic value of lymph node status and LNR.

\section{Methods}

\section{Patient Cohort}

Between 2009 and 2014, 83 patients with gallbladder carcinoma underwent laparotomy at Mahavir Cancer Institute and Research Centre. Twenty-three of them were deemed to be either metastatic or unresectable. Rest 60 patients were reviewed retrospectively in whom radical cholecystectomy was done for gallbladder carcinoma (Fig. 1).

Demographic profile, surgical, and pathological data were recorded.

The choice of the radicality of the procedure depends on the local extent of the tumor. Less aggressive resection was needed for early disease. The cases were followed up till July 2019, resulting in a follow-up period of $\geq 5$ years or till the death of the patient. No case was censored during survival analysis.

\section{Surgical Records}

The operation would consist of lymphadenectomy of the regional nodes, cholecystectomy, and about $2-\mathrm{cm}$ nonanatomical wedge resection of gallbladder fossa, to attain the negative surgical margin. Lymphadenectomy included removal of nodes along the common hepatic artery, hepatic artery proper, cystic duct, common bile duct (CBD), and portal vein. CBD resection was performed if the cystic duct margin came out to be positive on the frozen section.

\section{Histopathology}

Type, grade, depth of invasion, LVI, and PNI of the tumor were reviewed. As per AJCC eighth edition, the pathological T stage was $\mathrm{T} 1$ in five, T2 in 40, T3 in 13, and T4 in 2 cases.

Fifty-two cases had adenocarcinoma, five had adenosquamous type, and three had squamous cell carcinoma.

Lymph nodes were dissected from the resected specimen by the operating surgeon and were segregated into separate containers as per the locoregional stations. The positive lymph nodes were divided into three groups as per the AJCC eighth edition: N0, no regional lymph node metastasis; $\mathrm{N} 1$, metastases of 1 to 3 nodes; and N2, metastases of $\geq 4$ nodes. The total count of nodes dissected and nodes with metastases were noted down for each patient. The LNR was calculated by dividing the total count of metastatic nodes by the total dissected nodes. Cases were segregated into three categories as per LNR: One category who did not have any nodal involvement $(L N R=0)$, the second category with $L N R \leq 0.5$, and third category with LNR $>0.5$. After doing the statistical analyses, we found that this cutoff value was most discriminating.

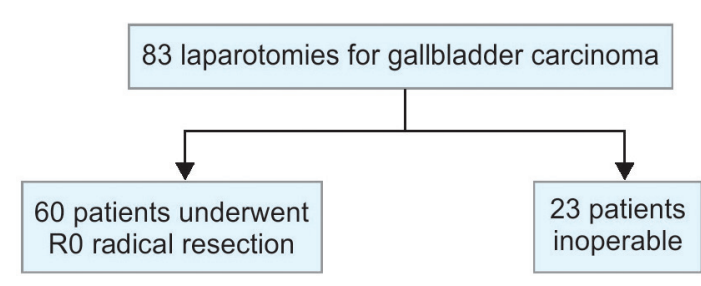

Fig. 1: Flowchart showing surgical management of all the patients with gallbladder cancer

\section{Follow-up}

We followed the patients for every 3 to 4 months for the first 2 years and every 6 months after that. We did clinical examination; tumor markers (CA19.0, CEA) and contrast-enhanced computed tomography were also done. If there was any suspicion of disease recurrence, we would do these tests earlier.

Adjuvant chemotherapy in the form of gemcitabine was given after resection if indicated. None of our patients received adjuvant radiotherapy.

We classified the recurrence as local or regional (liver bed or regional lymph nodes) and distant or both of these. Disease-free survival (DFS) was measured from the time of surgery to the time when recurrence of disease was diagnosed. The death of the patient was taken as due to the disease or because of some other causes so that disease-specific survival could be calculated.

\section{Statistical Analysis}

Data were collected for 60 patients. Continuous data are presented as median. Categorical variables were compared using Chi-square test. Survival was calculated from diagnosis to death. The primary outcome was DFS and is measured as median \pm standard error. Kaplan-Meier method was used for estimating survival. Cox proportional hazards regression model was used to calculate significant influence on survival. Multivariate logistic regression was used to determine the risk factors associated with disease recurrence. $p<0.05$ was considered statistically significant.

\section{Results}

The clinical attributes of our cases are presented in Table 1. At a median follow-up of 24 months, the overall DFS rate of the 60 patients at 3 years was $51 \%$ and at 5 years was 36\% (Fig. 2). The overall survival (OS) rate was $60 \%$ at 3 years and at 5 years was $42 \%$. There was no mortality in the postoperative period. The median DFS was 16.2 months, and OS was 21 months.

Forty cases (66.67\%) were node-negative who had a DFS of $62 \%$ at 3 years and $43 \%$ at 5 years (Fig. 2). The positive lymph nodes were found in 20 patients (33.34\%) who had a DFS of $30 \%$ at 3 years and $20 \%$ at 5 years (Fig. 2). Seventeen patients fell in the pN1 group, and three patients were in pN2 category. The mean number of harvested nodes was 6.6, and positive lymph nodes were 0.83 .

\section{Factors Affecting Disease-free Survival in Cases after Radical Cholecystectomy}

Median DFS was 16.6 months in 60 patients.

On univariate analysis, we found the factors associated with poor DFS were non-well-differentiated group, T-stage T3 or more, presence of LVI and PNI, lymph node metastases, higher $\mathrm{N}$ stage, and increased LNR (Table 2). On multivariate analysis, it was found that higher T-stage and higher LNR still were significant factors (Table 3). Cases that had T3 and T4 diseases had a median survival of only 6 months as opposed to the median survival of 20 months for cases in the T1 and T2 category. The T3 and T4 group cases had about 2 1/2-fold more risk of recurrence (HR, 2.98; 95\% Cl, 1.38-6.54; $p=0.005)$ when compared with cases in the T1 and T2 category. The LNR was strongly related to prognosis. Cases with a LNR of more than 0.5 had an about five-fold increased risk of recurrence (HR, 5.98; 95\% Cl, 2.566-13.961; $p<0.005)$ when compared with cases who had a LNR of 0 (Table 3 ). 
Table 1: Clinical characteristics of our patients

\begin{tabular}{lc}
\hline Clinical characteristics & $n(\%)$ \\
\hline Age in years & $29(48.33)$ \\
$<60$ & $31(51.66)$ \\
$>60$ & \\
Gender & $29(48.33)$ \\
Male & $31(51.66)$ \\
Female & $52(86.66)$ \\
Symtomatic & $25(48)$ \\
Pain & $5(9.6)$ \\
Jaundice & $22(42.3)$ \\
Postprandial fullness & $8(13.33)$ \\
Asymptomatic & \\
Differentiation & $27(45)$ \\
Well-differentiated & $33(55)$ \\
Others & \\
T stage & $39(65)$ \\
T1b/T2 & $21(35)$ \\
T3/T4 & \\
N stage & $40(66.66)$ \\
N0 & $17(28.33)$ \\
N1 & $3(5)$ \\
N2 & \\
LVI & $14(23.33)$ \\
Positive & $46(76.66)$ \\
Negative & \\
TLNC & $45(75)$ \\
$<6$ & $15(25)$ \\
\hline & \\
\hline & \\
\hline &
\end{tabular}

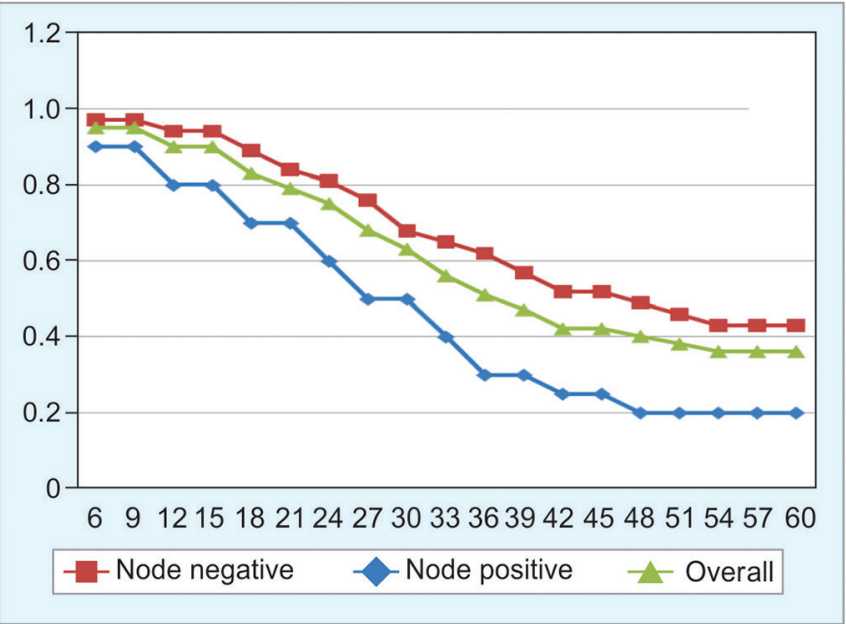

Fig. 2: Kaplan-Meier survival curves as per the presence or absence of regional nodal disease. Five-year DFS rate was $43 \%$ in patients without the regional nodal disease. The 5 -year DFS rate was $20 \%$ in patients with the regional nodal disease $(p<0.001)$.

\section{Factors Affecting Disease-free Survival in Patients with Stage IIIB Gallbladder Carcinoma after Radical Resection}

Stage IIIB cases include all N1 diseases with T1 to T3 in gallbladder carcinoma. In our study, we evaluated prognostic factors in
Table 2: Univariate analysis of factors associated with DFS after curativeintent resection for gallbladder cancer

\begin{tabular}{|c|c|c|c|}
\hline Variable & Patients & Recurrence & $p$ value \\
\hline Age (year) & & & NS \\
\hline$<60$ & 29 & $9(31.03)$ & \\
\hline$\geq 60$ & 31 & $12(38.7)$ & \\
\hline Sex & & & NS \\
\hline Male & 29 & $9(31.03)$ & \\
\hline Female & 31 & $12(38.7)$ & \\
\hline Symptom & & & NS \\
\hline Yes & 52 & $19(36.5)$ & \\
\hline No & 8 & $2(25)$ & \\
\hline Postoperative complication & & & NS \\
\hline Yes & 12 & $4(33.33)$ & \\
\hline No & 48 & $17(35.41)$ & \\
\hline LVI & & & $<0.05$ \\
\hline Yes & 14 & $7(50)$ & \\
\hline No & 46 & $14(30.4)$ & \\
\hline PNI & & & $<0.05$ \\
\hline Yes & 12 & $6(50)$ & \\
\hline No & 48 & $15(31.25)$ & \\
\hline Differentiation & & & $<0.05$ \\
\hline Well & 27 & $10(37.03)$ & \\
\hline Etc & 33 & $11(33.33)$ & \\
\hline T stage & & & $<0.05$ \\
\hline T1-T2 & 39 & $8(20.51)$ & \\
\hline T3-T4 & 21 & $13(61.9)$ & \\
\hline Lymph node involvement & & & $<0.05$ \\
\hline Yes & 14 & $9(64.28)$ & \\
\hline No & 46 & $12(26.08)$ & \\
\hline N stage & & & $<0.05$ \\
\hline No & 40 & $6(15)$ & \\
\hline N1 & 17 & $12(70.58)$ & \\
\hline N2 & 3 & $3(100)$ & \\
\hline Lymph node ratio (LNR) & & & $<0.05$ \\
\hline $\mathrm{LNR}=0$ & 40 & $6(15)$ & \\
\hline $0<$ LNR $<0.5$ & 11 & $6(54.5)$ & \\
\hline $0.5 \leq \mathrm{LNR} \leq 1.0$ & 9 & $9(100)$ & \\
\hline
\end{tabular}

Table 3: Multivariate analysis of factors associated with DFS after curative-intent resection for gallbladder cancer

\begin{tabular}{llll}
\hline Variable & Hazard ratio & $95 \% \mathrm{Cl}$ & p value \\
\hline T stage & & & 0.005 \\
T1-T2 & 1.0 & & \\
T3-T4 & 2.98 & $1.38-6.54$ & \\
Lymph node ratio (LNR) & & & $<0.005$ \\
LNR $=0$ & 1.0 & & \\
$0<\mathrm{LNR}<0.5$ & 1.09 & $0.315-3.654$ & \\
$0.5 \leq \mathrm{LNR} \leq 1.0$ & 5.98 & $2.566-13.961$ & \\
\hline
\end{tabular}

node-positive cases of T1 to T3 diseases, that is, stage IIIB patients. On univariate analysis, the total number of metastatic nodes and higher LNR was clearly connected with significantly worse DFS in node-positive patients. However, multivariate analysis showed only 
Table 4: Factors predictive of DFS in patients with stage IIIB gallbladder cancer after radical resection

\begin{tabular}{|c|c|c|c|c|}
\hline Variable & Patients & Recurrence & $\begin{array}{l}\text { Univariate } \\
\text { analysis } \\
\text { p value }\end{array}$ & $\begin{array}{l}\text { Multivariate } \\
\text { analysis } \\
H R(95 \% \text { CI) } \\
p \text { value }\end{array}$ \\
\hline Positive nodes & & & $<0.05$ & NS \\
\hline 1 & 7 & 6 & & \\
\hline $2-3$ & 11 & 7 & & \\
\hline$\geq 4$ & 2 & 2 & & \\
\hline LNR & & & $<0.05$ & $<0.05$ \\
\hline $0<\mathrm{LNR}<0.5$ & 11 & 6 & & \\
\hline $0.5 \leq \mathrm{LNR} \leq 1.0$ & 9 & 9 & & \\
\hline
\end{tabular}

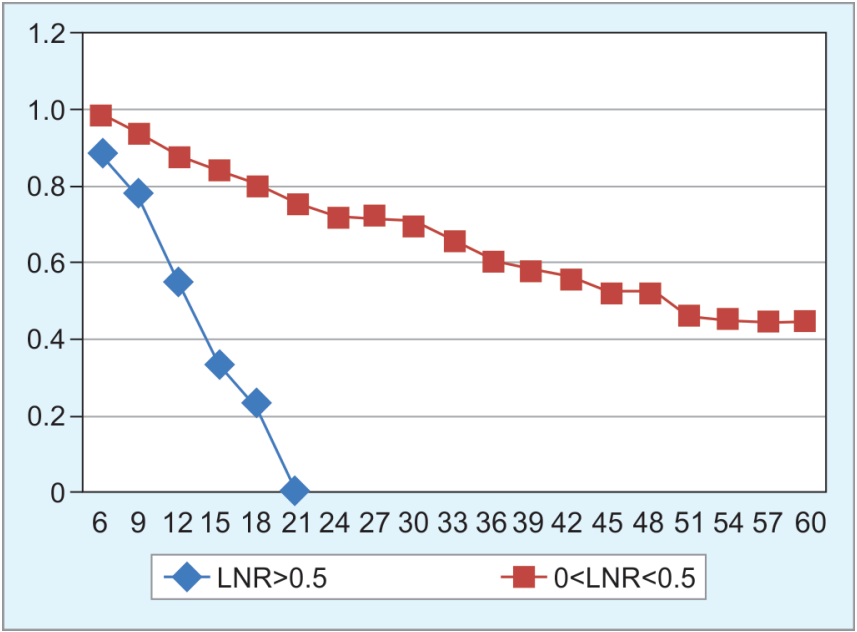

Fig. 3: Kaplan-Meier survival curves according to the LNR in patients with stage IIIB gallbladder cancer. Five-year DFS rate was $44 \%$ in patients with an LNR $<0.5$, but the median survival time was not reached. The 5 -year DFS rate was only $10 \%$ with a median survival time of 8.4 months in patients with a LNR $>0.5(p<0.005)$

higher LNR to be an independent prognostic factor of DFS in these cases (Table 4). Five-year DFS was $44 \%$ in cases who had LNR $<0.5$ (Fig. 3). The 5 -year DFS was only $10 \%$ with a median survival time of 8.4 months for cases with a LNR $>0.5$ (Fig. 3).

\section{Discussion}

Surgical resection to Ro probably is the only curative option in those patients who have resectable gallbladder cancer. In cases where they are present early, that is, there is involvement up to muscularis propria layer, good survival with radical resection has been seen. ${ }^{13-15}$ Simple cholecystectomy suffices for gallbladder carcinoma when tumor depth is only up to the lamina propria (T1a). Still, there is a debate about simple cholecystectomy versus extended cholecystectomy for the management of gallbladder carcinoma extending to the muscularis propria layer (T1b). Better survival has been reported in advanced gallbladder cancer after Glenn and Hays introduced radical cholecystectomy that includes en-bloc resection of gall bladder bed with a wedge of liver and nodes in a hepatoduodenal ligament. ${ }^{16}$ In locally advanced gallbladder carcinoma, extended resections have been performed like hepatectomies, pancreatoduodenectomy, and vascular resections. ${ }^{17}$ The two most common staging systems used to stage gallbladder carcinoma are: the eighth $\mathrm{AJCC}^{12}$ and the Japanese Society of Biliary Surgery system. ${ }^{3}$

Our study showed that the gallbladder carcinoma patients who had nodal metastases had poor survival as compared to those of node-negative disease; though on multivariate analysis, node positivity did not show any significant effect on DFS. Nodal metastasis is a strong indicator of worse prognosis in patients who have advanced carcinoma of gallbladder. ${ }^{18}$ Japanese data revealed that the survival of patients is improved in those cases who had lymph node involvement restricted to the nodes in the hepatoduodenal ligament and who undergo lymph node dissection. ${ }^{19}$ Our data demonstrated 5 -year survival of those cases who did not have any nodal metastases was $43 \%$ and that of those cases where there was nodal disease was $20 \%$ ( $p<0.001$ ). Among those cases who had nodal metastases, the N2 stage falls in stage IVB according to the eighth AJCC and has very meager long-term survival chances. In comparison, cases of $\mathrm{N} 1$ nodal disease, that is, stage IIIB, usually have longer survival. So, it becomes important to choose long-term surviving patients within stage IIIB and to identify the prognostic factors.

Recently published data have established the effect of total lymph nodes dissected, metastatic lymph nodes, and LNR on the survival of gallbladder carcinoma patients. ${ }^{20,21} \mathrm{TNrM}$ may be better than the TNM staging system for analyzing gallbladder carcinoma patients with less than six lymph nodes dissected, and it can supplement TNM staging for more precise risk categorization. ${ }^{22}$

In our study, the survival was shorter in patients with no nodal disease, that is, NO disease but less than six nodes dissected as compared to the survival of NO cases who had at least six lymph nodes dissected. Also, in their research, Negi et al. ${ }^{20}$ showed that patients with gallbladder carcinoma with N0 disease who had less than six lymph nodes dissected had a worse prognosis. It appears that total lymph nodes dissected in N0 patients are an important prognostic factor. We are of the opinion that those patients who have NO disease but less than six lymph nodes dissected are under stage due to inadequate lymph nodal dissection. Although there is no statistical significance in our research, a positive tendency was seen in this study, and in the paper published by Negi et al., ${ }^{20}$ there is a plausibility of this idea based on other site malignancies.

As opposed to NO cases, the total number of nodes dissected was not a significant prognostic factor in N1 cases. Probably the reason for it seems to be that the nodal metastases were a very strong prognostic factor that exceeded any prognostic effect of the total number of lymph nodes dissected.

If only the total number of affected lymph nodes is used as is in AJCC, it will lead to bias due to the chances of inadequate lymph nodal dissection. Many research papers have demonstrated LNR as an important prognostic factor for various gastrointestinal tumors. LNR, instead of a total number of lymph nodes dissected or a total number of metastatic lymph nodes, has an advantage due to various reasons. First of all, LNR is less susceptible to the phenomenon of stage migration. There are data available that have shown the superiority of LNR for gastric carcinoma as opposed to the usual staging of lymph nodes because it leads to stage migration. ${ }^{23}$ Additionally, comparison between the cases on the basis of lymph nodes involved may not be as accurate as LNR because LNR helps to provide a more practical comparison between cases with the same number of positive lymph nodes. As a sample, cases with three lymph nodes involved but the number of dissected lymph nodes are different (6 and 60) have different LNR 
(0.5 and 0.05). This example shows that the comparison between these two cases only on the basis of positive lymph node counts may not be correct. As such, it is better to consider LNR as a more practical variable to classify cases according to the extent of nodal involvement as well as appropriate lymph node dissection.

The data from our institute show LNR and T-stage variables influence DFS in cases who have undergone radical resection for gallbladder carcinoma irrespective of what the $\mathrm{N}$ stage is; particularly, a LNR more than 0.5 has the highest HR. In nodepositive cases, that is, N1, which fall in stage IIIB cases of gallbladder carcinoma, our data show that LNR was the strongest predictor of prognosis when we adjusted other competing factors, such as differentiation, LVI/PNI, T stage, number of metastatic nodes, and postoperative chemotherapy. Therefore, LNR was shown to be an independent prognostic factor in gallbladder carcinoma, particularly in N1 patients, in stage IIIB gallbladder carcinoma.

\section{Conclusion}

Lymph nodes dissected are a key prognostic factor in gallbladder carcinoma cases who underwent radical resection. The total number of lymph nodes dissected might have the impact on prognosis, particularly in node-negative, that is, N0 patients. In cases with node-positive T1 to T3 disease, that is, stage IIIB disease, a high LNR was shown as an accurate prognostic factor than the number of lymph nodes involved by metastases. LNR turned out to be a strong predictor of DFS despite controlling competing risk factors, particularly in stage IIIB patients. LNR has appeared as a strong prognostic factor and can be used as a classifying tool in further research evaluating adjuvant treatments.

\section{Contributions}

(I) Conception and design: Shah Naveed, Cao M Thau, and BB Panday; (II) Administrative support: None; (III) Provision of study materials or patients: BB Panday, Shah Naveed, and Hasina Qari; (IV) Collection and assembly of data: Hasina Qari, Pipit Burasakarn, and Abdul W Mir; (V) Data analysis and interpretation: Cao M Thau, Abdul W Mir, Shah Naveed, and Hasina Qari; (VI) Manuscript writing: All authors; (VII) Final approval of manuscript: All authors.

\section{References}

1. Justo I, Marcacuzco A, Nutu OA, et al. A retrospective analysis of patients with gallbladder cancer: surgical treatment and survival according to tumor stage. Rev Esp Enferm Dig 2018;110:485-492. DOI: $10.17235 /$ reed.2018.5435/2017.

2. Shirai Y, Wakai T, Hatakeyama K. Radical lymph node dissection for gallbladder cancer: indications and limitations. Surg Oncol Clin N Am 2007;16(1):221-232. DOI: 10.1016/j.soc.2006.10.011.

3. Japanese Society of Biliary Surgery. Classification of biliary tract carcinoma. 2nd ed. Tokyo: Kanehara; 2004.4.

4. Benoist S, Panis Y, Fagniez PL. French University Association for Surgical Research. Long-term results after curative resection for carcinoma of the gallbladder. Am J Surg 1998;175(2):118-122. DOI: 10.1016/s0002-9610(97)00269-9.

5. Tsukada K, Kurosaki I, Uchida K, et al. Lymph node spread from carcinoma of the gallbladder. Cancer 1997;80:661-667. PMID: 9264348
6. Endo I, Shimada H, Tanabe M, et al. Prognostic significance of the number of positive lymph nodes in gallbladder cancer. J Gastrointest Surg 2006;10(7):999-1007. DOI: 10.1016/j.gassur.2006.03.006.

7. Smith DD, Schwarz RR, Schwarz RE. Impact of total lymph node count on staging and survival after gastrectomy for gastric cancer: data from a large US-population database. J Clin Oncol 2005;23(28):71147124. DOI: 10.1200/JCO.2005.14.621.

8. Le Voyer TE, Sigurdson ER, Hanlon AL, et al. Colon cancer survival is associated with increasing number of lymph nodes analyzed: a secondary survey of intergroup trial INT-0089. J Clin Oncol 2003;21(15):2912-2919. DOI: 10.1200/JCO.2003.05.062.

9. Nitti $D$, Marchet $A$, Olivieri $M$, et al. Ratio between metastatic and examined lymph nodes is an independent prognostic factor after D2 resection for gastric cancer: analysis of a large European monoinstitutional experience. Ann Surg Oncol 2003;10(9):1077-1085. DOI: 10.1245/aso.2003.03.520.

10. Berger AC, Sigurdson ER, LeVoyer T, et al. Colon cancer survival is associated with decreasing ratio of metastatic to examined lymph nodes. J Clin Oncol 2005;23(34):8706-8712. DOI: 10.1200/ JCO.2005.02.8852.

11. Choi BG, Kim CY, Cho SH, et al. Impact of lymph node ratio as a valuable prognostic factor in gallbladder carcinoma, focusing on stage IIIB gallbladder carcinoma. J Korean Surg Soc 2013;84(3):168177. DOI: 10.4174/jkss.2013.84.3.168.

12. Edge SB, Mahul BA, Frederick LG. American Joint Committee on Cancer (AJCC) Staging Handbook: From the AJCC Cancer Staging Manual. 8th ed. Atlanta, GA: American Cancer Society; 2016.

13. Shimada $\mathrm{H}$, Endo $\mathrm{I}$, Togo $\mathrm{S}$, et al. The role of lymph node dissection in the treatment of gallbladder carcinoma. Cancer 1997;79(5):892-899. DOI: 10.1002/(sici)1097-0142(19970301)79:5<892::aid-cncr4>3.0.co;2-e.

14. Yamaguchi K, Chijiiwa K, Saiki S, et al. Retrospective analysis of 70 operations for gallbladder carcinoma. Br J Surg 1997;84(2):200-204. https://doi.org/10.1046/j.1365-2168.1997.02498.x

15. Miyakawa S, Ishihara $S$, Horiguchi $A$, et al. Biliary tract cancer treatment: 5,584 results from the Biliary Tract Cancer Statistics Registry from 1998 to 2004 in Japan. J Hepatobiliary Pancreat Surg 2009;16(1):1-7. DOI: 10.1007/s00534-008-0015-0.

16. Glenn F, Hays DM. The scope of radical surgery in the treatment of malignant tumors of the extrahepatic biliary tract. Surg Gynecol Obstet 1954;99(5):529-541. PMID : 13205425

17. Kondo S, Nimura Y, Hayakawa N, et al. Extensive surgery for carcinoma of the gallbladder. Br J Surg 2002;89(2):179-184. DOI: 10.1046/j.00071323.2001.02001.x.

18. Jensen EH, Abraham A, Jarosek $S$, et al. Lymph node evaluation is associated with improved survival after surgery for early stage gallbladder cancer. Surgery 2009;146(4):706-711. DOI: 10.1016/ j.surg.2009.06.056.

19. Kondo S, Takada T, Miyazaki M, et al. Guidelines for the management of biliary tract and ampullary carcinomas: surgical treatment. J Hepatobiliary Pancreat Surg 2008;15(1):41-54. DOI: 10.1007/s00534007-1279-5.

20. Negi SS, Singh A, Chaudhary A. Lymph nodal involvement as prognostic factor in gallbladder cancer: location, count or ratio? J Gastrointest Surg 2011;15(6):1017-1025. DOI: 10.1007/s11605 -011-1528-4.

21. Shirai Y, Sakata J, Wakai T, et al. Assessment of lymph node status in gallbladder cancer: location, number, or ratio of positive nodes. World J Surg Oncol 2012;10:87. DOI: 10.1186/1477-7819-10-87.

22. Li J, Sun Y, Zhao B, et al. Lymph node ratio-based staging system for gallbladder cancer with fewer than six lymph nodes examined. Front Oncol 2020;10:542005. DOI: 10.3389/fonc.2020.542005.

23. Bando $E$, Yonemura $Y$, Taniguchi K, et al. Outcome of ratio of lymph node metastasis in gastric carcinoma. Ann Surg Oncol 2002;9:775784. DOI: 10.1007/BF02574500. 